\title{
Search for Dark Matter with the Fermi Large Area Telescope
}

\author{
Aldo Morselli ${ }^{* \dagger}$ \\ INFN Roma Tor Vergata \\ E-mail: aldo.morsellieroma2.infn.it
}

Can we learn about New Physics with astronomical and astro-particle data? Since its launch in 2008, the Large Area Telescope, onboard of the Fermi Gamma-ray Space Telescope, has detected the largest amount of gamma rays in the $20 \mathrm{MeV} 300 \mathrm{GeV}$ energy range and electrons + positrons in the $7 \mathrm{GeV}-1 \mathrm{TeV}$ range. These impressive statistics allow one to perform a very sensitive indirect experimental search for dark matter. I will present the latest results on these searches and the comparison with LHC searches.

36th International Conference on High Energy Physics,

July 4-11, 2012

Melbourne, Australia

*Speaker.

$\dagger$ 


\section{Introduction}

The Fermi Observatory carries two instruments on-board: the Gamma-ray Burst Monitor (GBM) [1] and the Large Area Telescope (LAT) [2]. The GBM, sensitive in the energy range between $8 \mathrm{keV}$ and $40 \mathrm{MeV}$, is designed to observe the full unocculted sky with rough directional capabilities (at the level of one to a few degrees) for the study of transient sources, particularly Gamma-Ray Bursts (GRBs). The LAT is a pair conversion telescope for photons above $20 \mathrm{MeV}$ up to a few hundreds of $\mathrm{GeV}$. The field of view is $\sim 2.4 \mathrm{sr}$ and LAT observes the entire sky every $\sim 3$ hours ( 2 orbits). These features makes the LAT a great instrument for dark matter (DM) searches.

\section{Indirect Dark Matter searches}

One of the major open issues in our understanding of the Universe is the existence of an extremely-weakly interacting form of matter, the Dark Matter (DM), supported by a wide range of observations including large scale structures, the cosmic microwave background and the isotopic abundances resulting from the primordial nucleosynthesis. Complementary to direct searches being carried out in underground facilities and at accelerators, the indirect search for DM is one of the main items in the broad Fermi Science menu. The word indirect denotes here the search for signatures of Weakly Interactive Massive Particle (WIMP) annihilation or decay processes through the final products (gamma-rays, electrons and positrons, antiprotons) of such processes. Among many other ground-based and space-borne instruments, the LAT plays a prominent role in this search through a variety of distinct search targets: gamma-ray lines, Galactic and isotropic diffuse gamma-ray emission, dwarf satellites, CR electrons and positrons.

\subsection{Galactic center}

The Galactic center (GC) is expected to be the strongest source of $\gamma$-rays from DM annihilation, due to its coincidence with the cusped part of the DM halo density profile [7], [8], [9]. A preliminary analysis of the data, taken during the first 11 months of the Fermi satellite operations is presented in [10], [11].

The diffuse gamma-ray backgrounds and discrete sources, as we know them today, can account for the large majority of the detected gamma-ray emission from the Galactic Center. Nevertheless a residual emission is left, not accounted for by the above models [10], [11].

Improved modeling of the Galactic diffuse model as well as the potential contribution from other astrophysical sources (for instance unresolved point sources) could provide a better description of the data. Analyses are underway to investigate these possibilities.

\subsection{Dwarf galaxies}

Dwarf satellites of the Milky Way are among the cleanest targets for indirect dark matter searches in gamma-rays. They are systems with a very large mass/luminosity ratio (i.e. systems which are largely DM dominated). The LAT detected no significant emission from any of such systems and the upper limits on the $\gamma$-ray flux allowed us to put very stringent constraints on the parameter space of well motivated WIMP models [12]. 

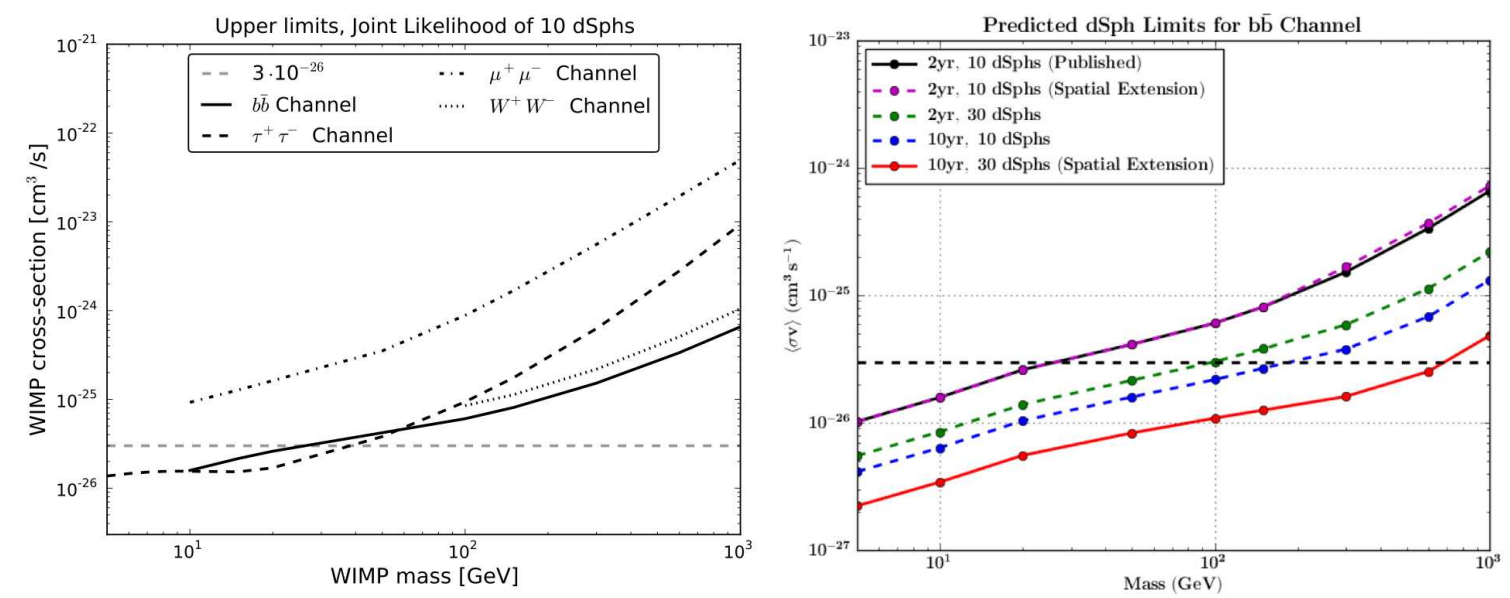

Figure 1: Derived 95\% C.L. upper limits on WIMP annihilation cross sections for different channels (left) and predicted 95\% C.L. upper limits on WIMP annihilation cross sections in 10 years for bbar channel

A combined likelihood analysis of the 10 most promising dwarf galaxies, based on 24 months of data and pushing the limits below the thermal WIMP cross section for low DM masses (below a few tens of $\mathrm{GeV}$ ), has been recently performed [14].

The derived 95\% C.L. upper limits on WIMP annihilation cross sections for different channels are shown in figure 1(left) The most generic cross section $\left(\sim 3 \cdot 10^{-26} \mathrm{~cm}^{3} \mathrm{~s}^{-1}\right.$ for a purely s-wave cross section) is plotted as a reference. These results are obtained for NFW profiles [13] but for cored dark matter profile the J-factors for most of the dSphs would either increase or not change much so these results includes J-factor uncertainties [14].

With the present data we are able to rule out large parts of the parameter space where the thermal relic density is below the observed cosmological dark matter density and WIMPs are dominantly produced non-thermally, e.g. in models where supersymmetry breaking occurs via anomaly mediation (see figure 2 for the MSSM model, updated from [12]).

These $\gamma$-ray limits also constrain some WIMP models proposed to explain the Fermi LAT and PAMELA $e^{+} e^{-}$data, including low-mass wino-like neutralinos and models with TeV masses pair-annihilating into muon-antimuon pairs.

Future improvements (apart from increased amount of data) will include an improved event selection with a larger effective area and photon energy range, and the inclusion of more satellite galaxies. In figures 1(right) and 2 are shown the predicted upper limits in the hypothesis of 10 years of data instead of 2; $30 \mathrm{dSphs}$ instead of ten (supposing that the new optical surveys will find new dSph); spatial extension analysis (source extension increases the signal region at high energy $E \geq 10 \mathrm{GeV}, M \geq 200 \mathrm{GeV}$ ).

Other complementary limits were obtained with the search of possible anisotropies generated by the DM halo substructures [15], the search for Dark Matter Satellites [16] or in the Galactic Halo [17] and a search for high-energy cosmic-ray electrons from the Sun [18].

\subsection{Gamma-ray lines}

A line at the WIMP mass, due to the $2 \gamma$ production channel, could be observed as a feature in 


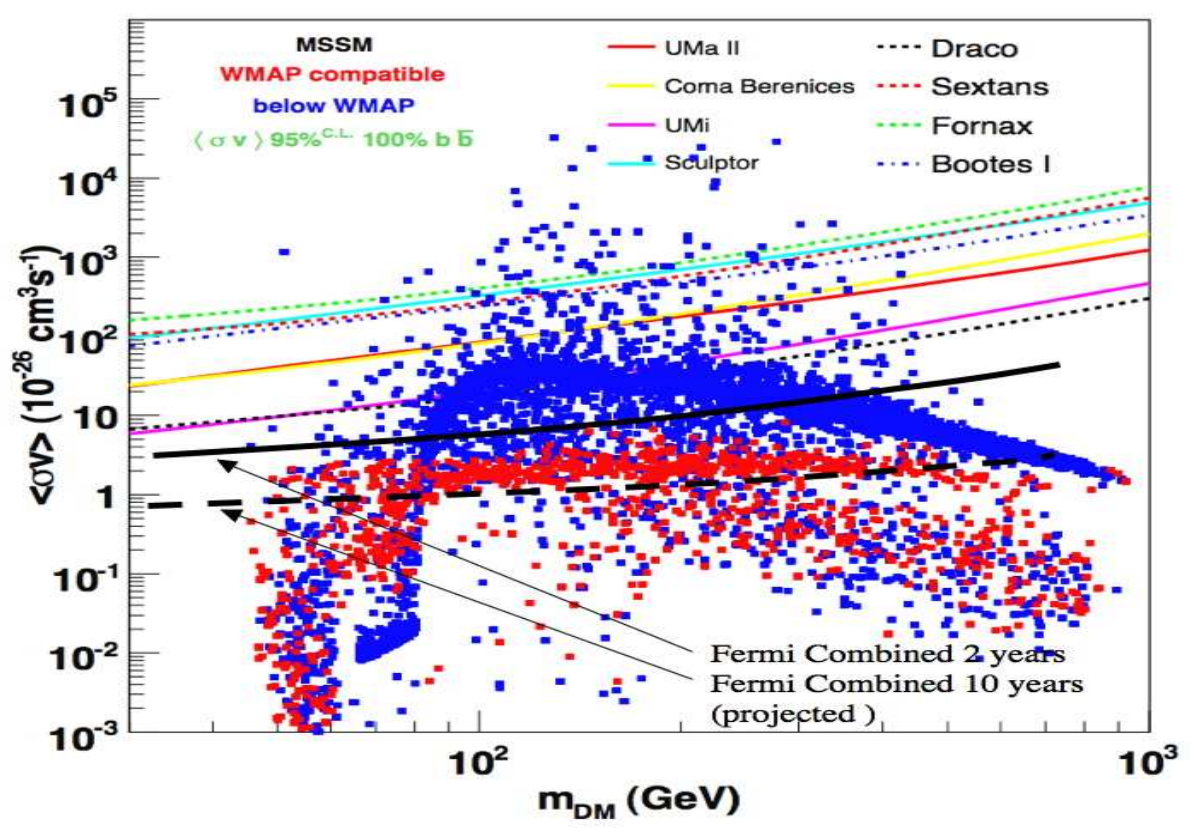

Figure 2: MSSM models in the $\left(m_{\mathrm{wimp}},<\sigma v>\right)$ plane. The models are consistent with all accelerator constraints and red points have a neutralino thermal relic abundance corresponding to the inferred cosmological dark matter density (blue points have a lower thermal relic density, and we assume that neutralinos still comprise all of the dark matter in virtue of additional non-thermal production processes

the astrophysical source spectrum [9]. Such an observation would be a "smoking gun" for WIMP $\mathrm{DM}$ as it is difficult to explain by a process other than WIMP annihilation or decay and the presence of a feature due to annihilation into $\gamma Z$ in addition would be even more convincing.

Up to now however no significant evidence of gamma-ray line(s) has been found in the first 11 months of data, between 30 and $200 \mathrm{GeV}$ [19] and in the first two years of from 7 to $200 \mathrm{GeV}$ [20] (see figure 3) and work is ongoing to extend the energy range of the analysis and include more data.

Recently, the claim of an indication of line emission in Fermi-LAT data [21, 22] has drawn considerable attention. Using an analysis technique similar to [19], but doubling the amount of data as well as optimizing the region of interest for signal over square-root of background, [21] found a (trial corrected) $3.2 \sigma$ significant excess at a mass of $\sim 130 \mathrm{GeV}$ that, if interpreted as a signal would amount to a cross-section of about $\left\langle\sigma v>\sim 10^{-27} \mathrm{~cm}^{3} \mathrm{~s}^{-1}\right.$.

The signal is concentrated on the Galactic Centre with a spatial distribution consistent with an Einasto profile [23]. This is marginally compatible with the upper limit presented in [20]. The main problems are the limited statistics in the GC sample and the check for any systematic effect that can mimic the line. A new version of the Instrument Response Function (IRF) (called Pass 8 ) is foreseen soon from the Fermi Lat collaboration. With this new analysis software we should increase the efficiency of the instrument at high energy and have a better control of the systematic effects. 


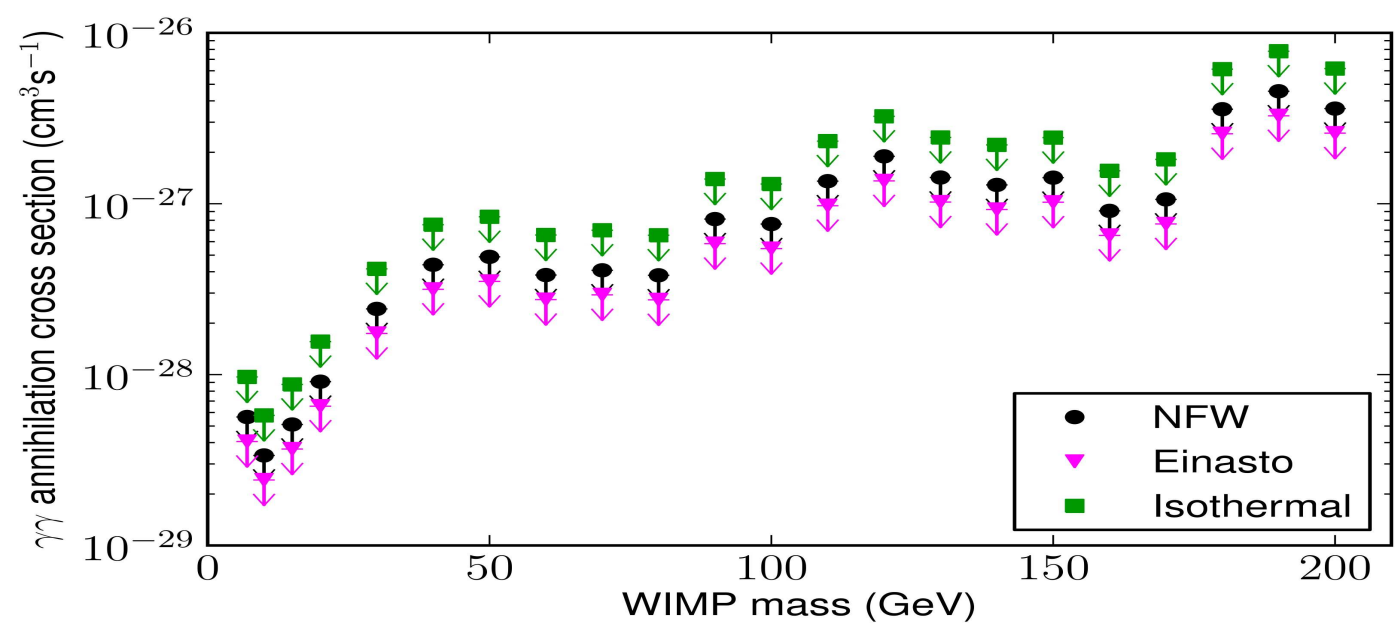

Figure 3: Dark matter annihilation 95\% CL cross section upper limits into $\gamma \gamma$ for the NFW, Einasto, and isothermal profiles for the region $|b|>10^{\circ}$ plus a $20^{\circ} \times 20^{\circ}$ square at the GC

\subsection{The Cosmic Ray Electron spectrum}

Recently the experimental information available on the Cosmic Ray Electron (CRE) spectrum has been dramatically expanded with a high precision measurement of the electron spectrum from 7 $\mathrm{GeV}$ to $1 \mathrm{TeV}$ [24], [25]. The spectrum shows no prominent spectral features and it is significantly harder than that inferred from several previous experiments.

More recently we provided a further, and stronger, evidence of the positron anomaly by providing direct measurement of the absolute $e^{+}$and $e^{-}$spectra, and of their fraction, between 20 and $200 \mathrm{GeV}$ using the Earth magnetic field. A steady rising of the positron fraction was observed up to that energy in agreement with that found by PAMELA. In the same energy range, the $e^{-}$ spectrum was fitted with a power-law with index $\gamma\left(e^{-}\right)=-3.19 \pm 0.07$ which is in agreement with what recently measured by PAMELA between 1 and $625 \mathrm{GeV}$ [26]. Most importantly, Fermi-LAT measured, for the first time, the $e^{+}$spectrum in the $20-200 \mathrm{GeV}$ energy interval (see figure 4) . The $e^{+}$spectrum is fitted by a power-law with index $\gamma\left(e^{+}\right)=-2.77 \pm 0.14$.

These measurements seems to rule out the standard scenario in which the bulk of electrons reaching the Earth in the $\mathrm{GeV}-\mathrm{TeV}$ energy range are originated by Supernova Remnants (SNRs) and only a small fraction of secondary positrons and electrons comes from the interaction of $\mathrm{CR}$ nuclei with the interstellar medium (ISM). An additional electron + positron component peaked at $\sim 1 \mathrm{TeV}$ seems necessary for a consistent description of all the available data sets. The temptation to claim the discovery of dark matter from detection of electrons from annihilation of dark matter particles is strong but there are competing astrophysical sources, such as pulsars, that can give a strong flux of primary positrons and electrons (see [29] and references therein). At energies between $100 \mathrm{GeV}$ and $1 \mathrm{TeV}$ the electron flux reaching the Earth may be the sum of an almost homogeneous and isotropic component produced by Galactic supernova remnants and the local contribution of a few pulsars with the latter expected to contribute more and more significantly as the energy increases. If a single pulsar give the dominant contribution to the extra component a large anisotropy and a small bumpiness should be expected; if several pulsars contribute the 


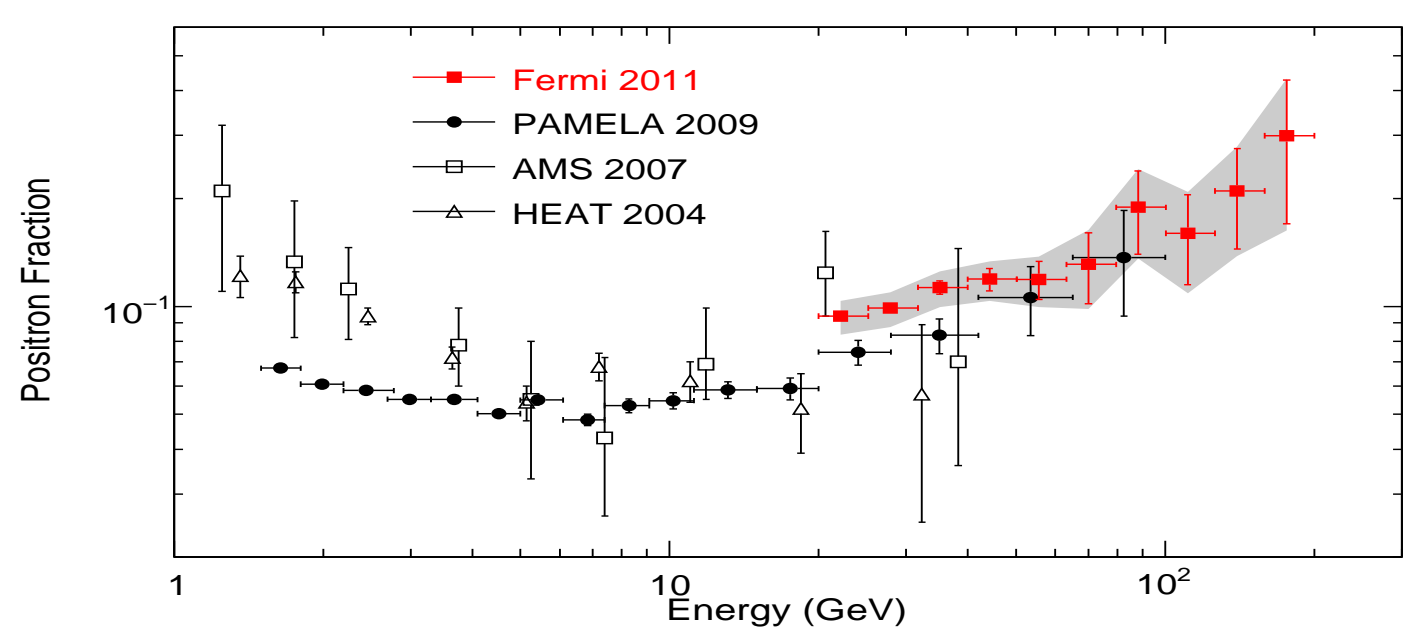

Figure 4: Positron fraction measured by the Fermi LAT and by other experiments [27, 28, 26]. The Fermi statistical uncertainty is shown with error bars and the total (statistical plus systematic uncertainty) is shown as a shaded band

opposite scenario is expected.

So far no positive detection of CRE anisotropy was reported by the Fermi-LAT collaboration, but some stringent upper limits were published [30] the pulsar scenario is still compatible with these upper limits.

Forthcoming experiments like AMS-02 and CALET are expected to reduce drastically the uncertainties on the propagation parameters by providing more accurate measurements of the spectra of the nuclear components of CR. Fermi-LAT and those experiments are also expected to provide more accurate measurements of the CRE spectrum and anisotropy looking for features which may give a clue of the nature of the extra component.

\section{Conclusions}

Fermi turned four years in orbit on June, 2012, and it is definitely living up to its expectations in terms of scientific results delivered to the community. The mission is planned to continue at least four more years (likely more) with many remaining opportunities for discoveries.

\section{Acknowledgments}

The Fermi LAT Collaboration acknowledges support from a number of agencies and institutes for both development and the operation of the LAT as well as scientific data analysis. These include NASA and DOE in the United States, CEA/Irfu and IN2P3/CNRS in France, ASI and INFN in Italy, MEXT, KEK, and JAXA in Japan, and the K. A. Wallenberg Foun- dation, the Swedish Research Council and the National Space Board in Sweden. Additional support from INAF in Italy and CNES in France for science analysis during the operations phase is also gratefully acknowledged. 


\section{References}

[1] Meegan, C.et al., 662, 469 (2003)

[2] W.B.Atwood et al. [Fermi Coll.] ApJ 697 No 2 (2009 June 1) 1071-1102 [arXiv:0902.1089]

[3] M.Ackermann et al. [Fermi Coll.] , Astroparticle Physics 35 (2012) $346 Đ 353$ [arXiv:1108.0201]

[4] M.Ackermann et al. [Fermi Coll.] , ApJS 203 (2012) 4 [arXiv:1206.1896]

[5] A.Abdo et al. [Fermi Coll.] ApJS 199, 31 (2012) [arXiv:1108.1435]

[6] A.Abdo et al.[Fermi Coll.] ApJS 188, 405 (2010) [arXiv:1002.2280]

[7] A. Morselli et al., Nucl.Phys. 113B (2002) 213

[8] A.Cesarini, F.Fucito, A.Lionetto, A.Morselli, P. Ullio, Astropart. Phys. 21 (2004) 267 [astro-ph/0305075]

[9] E. Baltz et al. , JCAP07 (2008) 013 [arXiv:0806.2911]

[10] V. Vitale and A. Morselli for the Fermi/LAT Collaboration, 2009 Fermi Symposium [arXiv:0912.3828]

[11] A. Morselli, B.Cañadas, V.Vitale, Il Nuovo Cimento Vol. 34 C, N. 3 (2011) [arXiv:1012.2292]

[12] A.Abdo et al. [Fermi Coll.], ApJ 712 (2010) 147-158 [arXiv:1001.4531]

[13] J.Navarro, J.Frenk, S.White Astrophys. J. 4621996563 [arXiv:astro-ph/9508025]

[14] M.Ackermann et al. [Fermi Coll.], 2011, Phys. Rev. Lett. 107, 241302 (2011) [arXiv:1108.3546]

[15] M.Ackermann et al. [Fermi Coll.], Phys. Rev. D 85, 083007 (2012) [arXiv:1202.2856]

[16] M.Ackermann et al. [Fermi Coll.], ApJ 747 (2012) 121 [arXiv:1201.2691]

[17] M.Ackermann et al. [Fermi Coll.], ApJ submitted [arXiv:1205.6474]

[18] M.Ajello et al. [Fermi Coll.], Phys. Rev. D 84, 032007 (2011) [arXiv:1107.4272]

[19] A.Abdo et al. [Fermi Coll.], Phys. Rev. Lett. 104, 091302 (2010) [arXiv:1001.4836]

[20] M.Ackermann et al. [Fermi Coll.], Physical Review D 86, (2012) 022002 [arXiv:1205.2739]

[21] C. Weniger, JCAP 1208 (2012) 007 [arXiv:1204.2797 [hep-ph]].

[22] M. Su and D. P. Finkbeiner, arXiv:1206.1616 [astro-ph.HE].

[23] T. Bringmann and C. Weniger, arXiv:1208.5481 [hep-ph]

[24] A.A.Abdoet al. [Fermi Coll.], PRL 102, 181101 (2009) [arXiv:0905.0025]

[25] M.Ackermann et al. [Fermi Coll.], Phys. Rev. D 82, 092004 (2010) [arXiv:1008.3999]

[26] O.Adriani. et al. [PAMELA Coll.], Phys. Rev. Lett. 106, 201101 ( 2011)

[27] M. A. DuVernois et al., [HEAT Coll.], ApJ 559, 296 (2001)

[28] M. Aguilar et al., [AMS Coll.], Physics Reports 366, 331 (2002)

[29] D. Grasso, S. Profumo, A. W. Strong, L. Baldini, R. Bellazzini, E. D. Bloom, J. Bregeon, G. di Bernardo, D. Gaggero, N. Giglietto, T. Kamae, L. Latronico, F. Longo, M. N. Mazziotta, A. A. Moiseev, A. Morselli, J. F. Ormes, M. Pesce-Rollins, M. Pohl, M. Razzano, C. Sgro, G. Spandre and T. E. Stephens, Astroparticle Physics (2009) 32, 140 [arXiv:0905.0636]

[30] M.Ackermann et al. [Fermi Coll.], Phys. Rev. D 82, 092003 (2010) [arXiv:1008.5119] 